\title{
Pacific
}

Journal of

Mathematics

\section{GENERIC FUNDAMENTAL POLYGONS FOR FUCHSIAN GROUPS}

AKIRA USHIJIMA 


\title{
GENERIC FUNDAMENTAL POLYGONS FOR FUCHSIAN GROUPS
}

\author{
AKIRA USHIJIMA
}

\begin{abstract}
A Dirichlet fundamental polygon for a Fuchsian group is said to be generic if its combinatorial shape is stable under any small permutation of the center of the polygon. Almost all points in the hyperbolic plane are known to be centers of generic fundamental polygons. We prove that the same property holds for points in the boundary of the hyperbolic plane.
\end{abstract}

\section{Introduction}

For a given topological space with a group action, a fundamental region is a subset consisting of representatives of the orbits of a given point by the action. In general, it is chosen to be connected. Such regions are used for the study of groups and their actions on spaces; they give tessellation of the spaces, which imply presentations of the groups.

When a metric is given to the space and the group is discrete, the Dirichlet domain (also known as the Voronoi cell) is an example of a fundamental region; for a point $p$ free under the group action, the Dirichlet domain for $p$ is the set of all points closer to $p$ than any other point in the orbit of $p$. For discrete groups acting on the hyperbolic plane, such domains are also called Dirichlet fundamental polygons with center $p$. We simply call them fundamental polygons in what follows.

One interesting question about fundamental polygons is how many different combinatorial shapes of such polygons are obtained from a given hyperbolic surface. This problem was considered for closed surfaces of genus two by Fricke and Klein [1897], and, independently, by Jørgensen and Näätänen [1982]. They showed that there were exactly eight types of "generic" fundamental polygons. Though the precise definition will be given in Section 3, a generic fundamental polygon has a property of stability of its combinatorial shape under any small perturbation of its center. Generic fundamental polygons are therefore in a sense far from the so-called canonical polygons of Fricke [Fricke and Klein 1897; Keen

This work was supported by a Grant-in-Aid for Young Scientists (B) 21740047, Japan.

MSC2000: primary 20H10; secondary 57M60.

Keywords: Fuchsian group, fundamental polygon. 
1966]. For closed surfaces of genus two, each generic fundamental polygon has 18 edges, while each canonical polygon has 8 edges.

Besides genus two, there are known facts about numbers of combinatorial shapes of admissible generic fundamental polygons for closed surfaces. The complete list of generic fundamental polygons of genus three was obtained in [Nakamura 2004]. Each such polygon has 30 edges. The formula to calculate possible numbers of combinatorial shapes of generic fundamental polygons for closed surfaces of any genus was obtained in [Bacher and Vdovina 2002]. Counting number of possible types is related to the study of extremal discs in a surface. For further results on this subject, see [Girondo and Nakamura 2007; Vdovina 2008].

Once we have known the number of combinatorial shapes of admissible generic fundamental polygons for a surface, it is also interesting to think about how these fundamental polygons are related to each other. Such a question was proposed in [Näätänen and Penner 1991] as follows: what kind of decomposition is given on a surface by a relation that two points on the surface are equivalent if they are centers of the fundamental polygon with the same combinatorial shape.

A local figure of such a decomposition of a closed surface of genus two was given in [Näätänen 1985]. In this figure, the set of points corresponding to nongeneric fundamental polygons seems to have measure zero. Beardon proved [1983, Theorem 9.4.5] that this is true for any Fuchsian group; for any given such group, almost all points in the hyperbolic plane are centers of generic fundamental polygons. A corresponding result for three-dimensional hyperbolic geometry, that is, for Kleinian groups, was proposed in [Jørgensen and Marden 1988]. However, the proof of Lemma 3.1 in that article, which plays an important role in the proof of the main result, is incomplete.

As a first try to give a complete proof of Jørgensen and Marden's result, we applied their strategy to the case for Fuchsian groups in [Díaz and Ushijima 2009]. We obtained an alternative proof of the result of Beardon there.

The idea of fundamental polygons can be generalized to the case where the center lies on the boundary of the hyperbolic plane. The main purpose of this paper is to show, again following the strategy of Jørgensen and Marden, that Beardon's result holds even when the centers lie in the boundary of the hyperbolic plane.

\section{Preliminaries}

Let $\mathbb{\boxplus}^{2}:=\{z \in \mathbb{C} \mid \operatorname{Im} z>0\}$ be the upper half-plane model of the two-dimensional hyperbolic space. It is given as a subset of the complex plane $\mathbb{C}$, but it is also regarded as contained in the Riemann sphere $\widehat{\mathbb{C}}:=\mathbb{C} \cup\{\infty\}$, where $\infty$ denotes the point at infinity. The boundary $\partial \mathbb{H}^{2}$ of $\mathbb{U}^{2}$ is considered in $\widehat{\mathbb{C}}$ so that it consists of

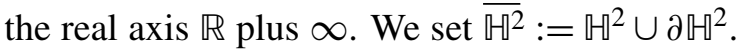


A circle in $\widehat{\mathbb{C}}$ means either a Euclidean circle in $\mathbb{C}$ or a Euclidean line in $\mathbb{C}$, as a circle through $\infty$. Hyperbolic lines in $\mathbb{U}^{2}$ (resp. in $\overline{\mathbb{U}^{2}}$ ) are obtained as the intersection of $\mathbb{U}^{2}$ (resp. $\overrightarrow{\mathbb{M}^{2}}$ ) and circles in $\widehat{\mathbb{C}}$ which are perpendicular to $\mathbb{R}$. For two distinct points $z$ and $w$ in $\overline{\mathbb{U}^{2}}$, we denote by $[z, w]$ the hyperbolic line segment with endpoints $z$ and $w$ in $\overline{\mathbb{M}^{2}}$.

The orientation-preserving isometry group of $\mathbb{U}^{2}$ is known to be isomorphic to the following projective special linear group:

$$
\operatorname{PSL}_{2}(\mathbb{R}):=\left\{\left(\begin{array}{ll}
a & b \\
c & d
\end{array}\right) \mid a, b, c, d \in \mathbb{R}, a d-b c=1\right\} /\{ \pm I\},
$$

where $I$ denotes the identity matrix. The action of an element $T$ in $\mathrm{PSL}_{2}(\mathbb{R})$ on $\widehat{\mathbb{C}}$ is a Möbius transformation

$$
T(z):=\frac{a z+b}{c z+d}, \quad z \in \widehat{\mathbb{C}} .
$$

The restriction of this action on $\mathbb{T}^{2}$ is orientation-preserving and isometric with respect to the hyperbolic metric. We denote the set of the fixed points of $T$ in $\overline{\mathbb{U}^{2}}$ by $\operatorname{Fix}(T)$. Any nontrivial element of $\mathrm{PSL}_{2}(\mathbb{R})$ is classified into three types according to the number of elements in $\operatorname{Fix}(T)$; a nontrivial element $T$ in $\mathrm{PSL}_{2}(\mathbb{R})$ is said to be elliptic if $\operatorname{Fix}(T)$ coincides with $\operatorname{Fix}(T) \cap \mathbb{H}^{2}$ that is a one point set, parabolic if $\operatorname{Fix}(T)$ coincides with $\operatorname{Fix}(T) \cap \partial \mathbb{U}^{2}$ that is a one point set, and hyperbolic if $\operatorname{Fix}(T)$ coincides with $\operatorname{Fix}(T) \cap \partial \mathbb{\boxplus}^{2}$ that consists of two points. For hyperbolic $T$, the axis $\operatorname{Ax}(T)$ is defined to be the hyperbolic line whose endpoints are the fixed points of $T$.

For an element $T$ in $\operatorname{PSL}_{2}(\mathbb{R})$ and a point $z$ in $\mathbb{U}^{2}-\operatorname{Fix}(T)$, let

$$
\mathrm{B}(z ; T):=\left\{w \in \mathbb{H}^{2} \mid d(w, z)=d(w, T(z))\right\}
$$

be the set of points in $\mathbb{H}^{2}$ that are equidistant from $z$ and $T(z)$ with respect to the hyperbolic distance $d(\cdot, \cdot)$. It is a hyperbolic line, the perpendicular bisector of $[z, T(z)]$. We remark that our definition of $\mathrm{B}(z ; T)$, as in [Díaz and Ushijima 2009], differs from the one given in [Jørgensen and Marden 1988]; there $\mathrm{B}(z ; T)$ was defined as the perpendicular bisector of $\left[z, T^{-1}(z)\right]$.

The definition of $\mathrm{B}(z ; T)$ generalizes to the case that the point $z$ lies in $\partial \mathbb{U}^{2}$. For a point $p$ in $\partial \mathbb{Q}^{2}-\operatorname{Fix}(T)$, a hyperbolic line $\mathrm{B}(p ; T)$ is defined to be the limit of $\mathrm{B}(z ; T)$ as $z$ converges to $p$. In particular, if $p$ is taken to be $\infty$, then $\mathrm{B}(\infty ; T)$ is the isometric semicircle for $T^{-1}$.

We denote by $\overline{\mathrm{B}(z ; T)}$ the closure of $\mathrm{B}(z ; T)$ in $\overline{\mathbb{U}^{2}}$. For further properties and a proof of the following proposition [Jørgensen and Marden 1988, Section 2].

Proposition 1. Let $T$ be a nontrivial element in $\mathrm{PSL}_{2}(\mathbb{R})$. 
(1) If $T$ is elliptic, then $\mathrm{B}(z ; T)$ contains the fixed point of $T$ for any point $z$ in $\overline{\mathbb{M}^{2}}-\operatorname{Fix}(T)$.

(2) If $T$ is parabolic, then $\overline{\mathrm{B}(z ; T)}$ contains the fixed point of $T$ for any point $z$ in $\overline{\mathbb{M}^{2}}-\operatorname{Fix}(T)$. If the point $z$ approaches the fixed point $\zeta$ of $T$ conically, then $\overline{\mathrm{B}(z ; T)}$ converges to $\zeta$.

(3) If $T$ is hyperbolic, then $\overline{\mathrm{B}(z ; T)}$ does not contain any fixed point of $T$ for any point $z$ in $\overline{\mathbb{W}^{2}}-\mathrm{Fix}(T)$. Furthermore it intersects perpendicularly with $\operatorname{Ax}(T)$. If the point $z$ approaches a fixed point $\zeta$ of $T$, then $\overline{\mathrm{B}(z ; T)}$ converges to $\zeta$.

Fuchsian groups are discrete subgroups of the orientation-preserving isometry group of $\mathbb{U}^{2}$. We regard them as subgroups of $\mathrm{PSL}_{2}(\mathbb{R})$ in what follows. For a given Fuchsian group $\Gamma$ and a point $w$ in $\mathbb{U}^{2}$, we define a subset $\mathscr{P}_{0}(w)$ in $\mathbb{H}^{2}$ as follows:

$$
\mathscr{P}_{0}(w):=\left\{z \in \mathbb{H}^{2} \mid d(z, w) \leq d(z, T(w)) \text { for all } T \in \Gamma\right\}
$$

when $w$ is in $\mathbb{T}^{2}$, and

$$
\mathscr{P}_{0}(w):=\left\{z \in \mathbb{Q}^{2} \mid \begin{array}{l}
\text { for any } T \in \Gamma-\{I\}, \text { the point } z \text { lies in the closure of } \\
\text { the component of } \mathbb{U}^{2}-\mathrm{B}(w ; T) \text { that is adjacent to } w
\end{array}\right\}
$$

when $w$ is in $\partial \mathbb{H}^{2}$. The subset $\mathscr{P}_{0}(w)$ is a fundamental polygon for $\Gamma$ when $w$ is taken from $\mathbb{R}^{2}-\bigcup_{T \in \Gamma} \operatorname{Fix}(T)$. Then $\mathscr{P}_{0}(w)$ is called a (Dirichlet) fundamental polygon for $\Gamma$. For a point $w$ in $\partial \mathbb{U}^{2}$, on the other hand, the subset $\mathscr{P}_{0}(w)$ is not always a fundamental polygon. Let $\Omega(\Gamma)$ be the ordinary set for $\Gamma$ in $\widehat{\mathbb{C}}$. It is shown in [Beardon 1983, Theorem 9.5.2] that $\mathscr{P}_{0}(w)$ is a fundamental polygon when $w$ is taken from $\Omega(\Gamma)$. When such $w$ is taken to be $\infty$, the fundamental polygon $\mathscr{P}_{0}(w)$ is known as the Ford fundamental region. Set $\mathscr{P}(w):=\overline{\mathscr{P}_{0}(w)} \cap \Omega(\Gamma)$, where $\overline{\mathscr{P}_{0}(w)}$ means the closure of $\mathscr{P}(w)$ in $\mathbb{\mathbb { M }}^{2}$. The point $w$ is called the center of $\mathscr{P}_{0}(w)$, or of $\mathscr{P}(w)$.

\section{Generic fundamental polygons and the main result}

Since the polygon $\mathscr{P}(w)$ is defined to be the intersection of $\overline{\mathscr{P}_{0}(w)}$ with $\Omega(\Gamma)$, the vertices of $\mathscr{P}_{0}(w)$, or the endpoints in $\Omega(\Gamma)$ of edges of $\mathscr{P}_{0}(w)$, are vertices of $\mathscr{P}(w)$. Fixed points of elliptic elements of order two on edges of $\mathscr{P}_{0}(w)$ are also called vertices of $\mathscr{P}(w)$. Vertices in $\mathbb{H}^{2}$ are called inner vertices, and those in $\Omega(\Gamma)$ are called boundary vertices.

A cusp of $\mathscr{P}(w)$ is a parabolic fixed point lying in $\overline{\mathscr{P}}(w)$. Cusps are not boundary vertices, since any parabolic fixed point belongs to the limit set, which is the complement of $\Omega(\Gamma)$ in $\widehat{\mathbb{C}}$.

The edges of $\mathscr{P}(w)$ are either those of $\mathscr{P}_{0}(w)$ or closed segments in $\mathscr{P}(w) \cap \partial \mathbb{H}^{2}$. An edge of $\mathscr{P}_{0}(w)$ as a hyperbolic polygon is decomposed into two edges of $\mathscr{P}(w)$ 
if it has a vertex corresponding to the fixed point of an elliptic element of order two. The edges of $\mathscr{P}(w)$ which come from those of $\mathscr{P}_{0}(w)$ are also called inner edges. We denote by $\ell(e)$ the hyperbolic line containing an inner edge $e$ of $\mathscr{P}_{0}(w)$.

An inner vertex $v$ of $\mathscr{P}(w)$ said to have a vertex cycle of length $k$ if there is a sequence $T_{1}=I, T_{2}, T_{3}, \ldots, T_{k}, T_{k+1}=T_{1}=I$ of elements in $\Gamma$ such that the sequence $T_{1}(\mathscr{P}(w))=\mathscr{P}(w), T_{2}(\mathscr{P}(w)), \ldots, T_{k}(\mathscr{P}(w))$ of polygons is a cyclic arrangement around $v$ in the $\Gamma$-orbit of $\mathscr{P}(w)$. In other words, the length of a vertex cycle is the number of disjoint vertices of $\mathscr{P}_{0}(w)$ that are equivalent to $v$ under $\Gamma$ if $v$ is not fixed by elliptic elements in $\Gamma$. The sequence $T_{1}, T_{2}, \ldots, T_{k}$ is called the vertex cycle of $v$.

Definition. For a Fuchsian group $\Gamma$, the fundamental polygon $\mathscr{P}(w)$ centered at $w$ in $\mathbb{H}^{2} \cup \Omega(\Gamma)$ is said to be generic if it satisfies the following conditions:

(1) For an inner vertex, if the length of its vertex cycle is greater than three, then the vertex is the fixed point of an elliptic element in $\Gamma$.

(2) For an inner edge $e$, if $\overline{\ell(e)}$ contains the fixed point of an elliptic or a parabolic element in $\Gamma$, the element of $\Gamma$ defining $\ell(e)$ as the bisector is an elliptic or a parabolic element fixing the point in question. Similarly, if $\ell(e)$ intersects perpendicularly with the axis of a hyperbolic element in $\Gamma$, the element of $\Gamma$ defining $\ell(e)$ as the bisector is a hyperbolic element fixing the axis in question.

(3) Every boundary vertex is an endpoint of exactly one inner edge.

(4) If two inner edges share an endpoint on $\partial \mathbb{Q}^{2}$, then the endpoint is a cusp that is the fixed point of a parabolic element gluing these inner edges.

Analogous notions have been studied before. Our definition of generic fundamental polygons is the two-dimensional counterpart of the definition of generic fundamental polyhedra in [Jørgensen and Marden 1988]. Our Conditions (1), (3), and (4) correspond to those defining Dirichlet polygons in [Beardon 1983, Theorem 9.4.5].

The conditions for generic fundamental polygons have geometric interpretations. Condition (1) and (2) together imply that, if a vertex $v$ is fixed by an elliptic element in $\Gamma$, its vertex cycle coincides with the cyclic elliptic subgroup with fixed point $v$. Another interpretation is that any cone singularity of the surface $\mathbb{M}^{2} / \Gamma$ is cut by the image of exactly one inner edge. Similarly, Conditions (2) and (3) mean that any border (open end) of $\mathbb{M}^{2} / \Gamma$ is also cut by the image of exactly one inner edge, and Condition (4) means that any cusp of $\mathbb{\boxplus}^{2} / \Gamma$ is also cut by the image of exactly one inner edge.

Theorem. For a Fuchsian group $\Gamma$, there is a subset $\mathcal{N}_{\Gamma}$ in $\partial \mathbb{H}^{2}$ of measure zero such that, for any point $w$ in $\left(\Omega(\Gamma) \cap \partial \mathbb{Q}^{2}\right)-\mathcal{N}_{\Gamma}$, the fundamental polygon $\mathscr{P}(w)$ is generic. 
Proof. To obtain the subset $\mathcal{N}_{\Gamma}$, we define three families of subsets in $\partial \mathbb{W}^{2}$. For $T_{1}, T_{2}, T_{3}$ in $\mathrm{PSL}_{2}(\mathbb{R})$, define a subset $\mathscr{V}_{T_{1}, T_{2}, T_{3}}$ in $\partial \mathbb{\boxplus}^{2}$ as

$$
\mathscr{V}_{T_{1}, T_{2}, T_{3}}:=\left\{p \in \partial \mathbb{T}^{2} \mid \overline{\mathrm{B}\left(p ; T_{1}\right)} \cap \overline{\mathrm{B}\left(p ; T_{2}\right)} \cap \overline{\mathrm{B}\left(p ; T_{3}\right)} \neq \varnothing\right\} .
$$

For $T_{1}, T_{2}$ in $\mathrm{PSL}_{2}(\mathbb{R})$, define $\mathscr{T}_{T_{1}, T_{2}}$ as

$$
\mathscr{T}_{T_{1}, T_{2}}:=\left\{p \in \partial \mathbb{H}^{2} \mid \overline{\mathrm{B}\left(p ; T_{1}\right)} \cap \overline{\mathrm{B}\left(p ; T_{2}\right)} \cap \partial \mathbb{M}^{2} \neq \varnothing\right\} .
$$

For $T_{1}, T_{2}$ in $\mathrm{PSL}_{2}(\mathbb{R})$, define $\mathscr{F}_{T_{1}, T_{2}}$ as

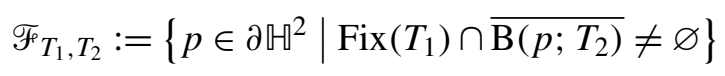

when $T_{1}$ is elliptic or parabolic, and

$$
{ }^{F_{F}} T_{1}, T_{2}:=\left\{p \in \partial \mathbb{T}^{2} \mid \mathrm{B}\left(p ; T_{2}\right) \text { intersects perpendicularly with the axis of } T_{1}\right\}
$$

when $T_{1}$ is hyperbolic.

Using these subsets, we define

$$
\mathcal{N}_{\Gamma}:=\left(\bigcup_{\left\{T_{1}, T_{2}, T_{3}\right\}} \mathscr{V}_{T_{1}, T_{2}, T_{3}}\right) \cup\left(\bigcup_{\left\{T_{4}, T_{5}\right\}} \mathscr{T}_{T_{4}, T_{5}}\right) \cup\left(\bigcup_{\left(T_{6}, T_{7}\right)} \mathscr{F}_{T_{6}, T_{7}}\right),
$$

where $\left\{T_{1}, T_{2}, T_{3}\right\}$ runs over all triples in $\Gamma$ that are mutually distinct, nontrivial and neither elliptic with a common fixed point nor parabolic with a common fixed point, $\left\{T_{4}, T_{5}\right\}$ runs over all pairs in $\Gamma$ that are distinct, nontrivial and not parabolic with a common fixed point, and $\left(T_{6}, T_{7}\right)$ runs over all ordered pairs of nontrivial elements in $\Gamma$ such that $\operatorname{Fix}\left(T_{7}\right)$ and $\operatorname{Fix}\left(T_{6}\right)$ are different.

Each of the indexes in the definition of $\mathcal{N}_{\Gamma}$ runs over countably many triples and pairs, because $\Gamma$ is a discrete group (see [Beardon 1983, Exercise 2.3.3], for example,). To see that $\mathcal{N}_{\Gamma}$ has measure zero, it is thus enough to see that the subsets $\mathscr{V}_{T_{1}, T_{2}, T_{3}}, \mathscr{T}_{T_{4}, T_{5}}$ and $\mathscr{F}_{T_{6}, T_{7}}$ have measure zero. These are shown in Propositions 5, 7 and 9 , respectively.

We next use case analysis to show that the fundamental polygon $\mathscr{P}(w)$ is generic for any $w$ in $\left(\Omega(\Gamma) \cap \partial \mathbb{H}^{2}\right)-\mathcal{N}_{\Gamma}$.

For Condition (1), take a vertex $v$ of $\mathscr{P}_{0}(w)$. Let $S_{v}$ be the vertex cycle of $v$, and $k_{v}$ the length of $S_{v}$. Suppose $k_{v}>3$. Then there are at least three nontrivial and distinct elements, say $T_{a}, T_{b}$ and $T_{c}$, in $S_{v}$. Though $w$ is not in $\bigcup_{\left\{T_{1}, T_{2}, T_{3}\right\}} \mathcal{V}_{T_{1}, T_{2}, T_{3}}$, the bisectors $\mathrm{B}\left(w ; T_{a}\right), \mathrm{B}\left(w ; T_{b}\right)$ and $\mathrm{B}\left(w ; T_{c}\right)$ contain $v$. This means that the three elements $T_{a}, T_{b}$ and $T_{c}$ are either elliptic with a common fixed point or parabolic with a common fixed point. If they are parabolic, then $\overline{\mathrm{B}\left(w ; T_{a}\right)}, \overline{\mathrm{B}\left(w ; T_{b}\right)}$ and $\overline{\mathrm{B}\left(w ; T_{c}\right)}$ contain a common fixed point in $\partial \mathbb{H}^{2}$. This contradicts the assumption that $v$ is in $\mathbb{H}^{2}$. If they are elliptic with a common fixed point, $S_{v}$ is in a cyclic subgroup of $\Gamma$ by the discreteness of $\Gamma$. Then the cyclic subgroup is generated by an elliptic element, which fixes $v$. 
For Condition (2), take an inner edge $e$ of $\mathscr{P}(w)$. Let $T_{e}$ be an element in $\Gamma$ such that $\ell(e)$ coincides with $\mathrm{B}\left(w ; T_{e}\right)$. Suppose that an endpoint of $\ell(e)$ coincided with the fixed point of a parabolic element, say $T$. This means that $\operatorname{Fix}(T) \cap \overline{\mathrm{B}\left(w ; T_{e}\right)}$ is nonempty. Since $w$ is not in $\bigcup_{\left(T_{6}, T_{7}\right)} \mathscr{F}_{T_{6}, T_{7}}$, the set $\operatorname{Fix}\left(T_{e}\right)$ coincides with $\operatorname{Fix}(T)$. The same argument is applied to the cases that $T_{e}$ is either elliptic or hyperbolic.

For Conditions (3), and (4), take a vertex (in the ordinary sense) $v^{*}$ of $\overline{\mathscr{P}(w)}$ that is in $\partial \mathbb{M}^{2}$. Suppose that $v^{*}$ is the endpoint of two inner edges, say $a$ and $b$, of $\mathscr{P}_{0}(w)$. Let $T_{a}$ and $T_{b}$ are elements in $\Gamma$ such that $\ell(a)$ and $\ell(b)$ coincide with $\mathrm{B}\left(w ; T_{a}\right)$ and $\mathrm{B}\left(w ; T_{b}\right)$ respectively. Since $w$ is not in $\bigcup_{\left\{T_{4}, T_{5}\right\}} \mathscr{T}_{T_{4}, T_{5}}$, the elements $T_{a}$ and $T_{b}$ are parabolic with a common fixed point. Then $v^{*}$ is their common fixed point so that it is a cusp of $\mathscr{P}(w)$ given as the endpoint of $a$ and $b$ that are glued together by $T_{a}=T_{b}{ }^{-1}$.

Furthermore, the argument above implies that a vertex $v^{*}$ in $\Omega(\Gamma)$ is an endpoint of exactly one inner edge, for any parabolic fixed point is not in $\Omega(\Gamma)$. Such a vertex is a boundary vertex by definition.

We have thus shown that $\mathscr{P}(w)$ is generic for any $w$ in $\left(\Omega(\Gamma) \cap \partial \mathbb{\boxplus}^{2}\right)-\mathcal{N}_{\Gamma}$.

This theorem is a generalization of [Beardon 1983, Theorem 9.4.5] and [Díaz and Ushijima 2009, Corollary 3.11]. The algebraic equations defining $\mathscr{V}_{T_{1}, T_{2}, T_{3}}$, $\mathcal{T}_{T_{4}, T_{5}}$ and $\mathscr{F}_{T_{6}, T_{7}}$ can be regarded as defined on $\mathbb{C}$, so the set $\mathcal{N}_{\Gamma}$ can be extended to one in $\overline{\mathbb{H}^{2}}$. Both $\mathcal{N}_{\Gamma}$ and its extension have measure zero, so their complements are dense in $\partial \mathbb{U}^{2}$ and $\overline{\mathbb{U}^{2}}$. The fundamental polygon $\mathscr{P}(w)$ is thus generic for almost every point $w$ in $\mathbb{H}^{2}$ and $\Omega(\Gamma) \cap \partial \mathbb{\boxplus}^{2}$.

\section{Propositions}

To prove the propositions used in the proof of the theorem, we use the projective disc model $D^{2}$ of two-dimensional hyperbolic space. As a set it is the open unit disc centered at the origin in two-dimensional real projective space $\mathbb{R} \mathbb{P}^{2}$. We choose the isometry from $\mathbb{U}^{2}$ to $D^{2}$ so that $0,1, \infty \in \partial \mathbb{U}^{2}$ are mapped, respectively, to $(0,-1),(1,0),(0,1)$ in $\mathbb{R} \mathbb{P}^{2}$.

Given an element $T=\left(\begin{array}{ll}a & b \\ c & d\end{array}\right)$ in $\operatorname{PSL}_{2}(\mathbb{R})$ and a point $z$ in $\overline{\mathbb{M}^{2}}-\mathrm{Fix}(T)$, let $\mathrm{C}(z ; T)$ be the pole in $\mathbb{R} \mathbb{P}^{2}$ of the projective line containing the image of $\mathrm{B}(z ; T)$ in $D^{2}$. For a point $p \in \mathbb{R} \subset \partial \mathbb{Q}^{2}$, a formula in [Jørgensen and Marden 1988, §2.9] tells us the coordinate of $\mathrm{C}(p ; T)$ :

$$
\begin{aligned}
\mathrm{C}(p ; T)= & \frac{1}{(a p+b)^{2}+(c p+d)^{2}-p^{2}-1} \\
& \quad \times\left(2((a p+b)(c p+d)-p),(a p+b)^{2}-(c p+d)^{2}-p^{2}+1\right) .
\end{aligned}
$$

Using this formula, we have the following proposition, which is a key in proving others. 
Proposition 2. For distinct and nontrivial elements $T_{1}, T_{2}$ and $T_{3}$ in $\mathrm{PSL}_{2}(\mathbb{R})$, the following two conditions are equivalent:

(1) The three points $\mathrm{C}\left(p ; T_{1}\right), \mathrm{C}\left(p ; T_{2}\right)$ and $\mathrm{C}\left(p ; T_{3}\right)$ are collinear in $\mathbb{R P}^{2}$ for any point $p$ in $\partial \mathbb{W}^{2}-\bigcup_{i=1}^{3} \operatorname{Fix}\left(T_{i}\right)$.

(2) The elements $T_{1}, T_{2}$ and $T_{3}$ either

(a) have the same fixed point set, or

(b) up to conjugation by an element of $\mathrm{PSL}_{2}(\mathbb{R})$, satisfy

$$
T_{1}(z)=a z, \quad T_{2}(z)=b z+1-b, \quad T_{3}(z)=\frac{a z}{(a-b) z+b},
$$

for some $a, b$ in $\mathbb{R}-\{0,1\}$.

Proof. This result is closely related to Theorem 4.3 in [Díaz and Ushijima 2009], which says that Condition (2) of the proposition holds if and only if, for any $z \in \mathbb{H}^{2}$, the points $z, T_{1}(z), T_{2}(z)$ and $T_{3}(z)$ are cocyclic in $\widehat{\mathbb{C}}$. We will see in Proposition 3 that this condition is equivalent to $\mathrm{C}\left(z ; T_{1}\right), \mathrm{C}\left(z ; T_{2}\right)$ and $\mathrm{C}\left(z ; T_{3}\right)$ being collinear for any $z \in \mathbb{H}^{2}-\bigcup_{i=1}^{3} \operatorname{Fix}\left(T_{i}\right)$. This shows the implication $(2) \Rightarrow(1)$.

Moreover the collinearity of $\mathrm{C}\left(z ; T_{1}\right), \mathrm{C}\left(z ; T_{2}\right)$ and $\mathrm{C}\left(z ; T_{3}\right)$ is an algebraic condition (see proof of the theorem just cited). If $T_{1}, T_{2}$ and $T_{3}$ are not one of the triples listed in Condition (2), the algebraic equation is proper, that is, the solution set is nowhere dense in $\mathbb{C}$. This, however, does not guarantee that $\mathbb{R}$ is not contained in the solution set. Thus the implication $(1) \Rightarrow(2)$ is not proved yet.

Let $T_{1}, T_{2}, T_{3}$ be distinct nontrivial elements of $\mathrm{PSL}_{2}(\mathbb{R})$ satisfying (1). Then the determinant $\Delta(p)$ of the $2 \times 2$ matrix with columns $\mathrm{C}\left(p ; T_{1}\right)-\mathrm{C}\left(p ; T_{2}\right)$ and $\mathrm{C}\left(p ; T_{1}\right)-\mathrm{C}\left(p ; T_{3}\right)$ vanishes wherever it is defined - that is, for any $p \in \mathbb{R}$ such that the points $\mathrm{C}\left(p ; T_{1}\right), \mathrm{C}\left(p ; T_{2}\right), \mathrm{C}\left(p ; T_{3}\right)$ are not on the line at infinity of $\mathbb{R} \mathbb{P}^{2}$. (Here of course the $\mathrm{C}\left(p ; T_{i}\right)$ are given by the formula $(*)$.)

Now, as $p$ runs over $\partial \mathbb{U}$, each $\mathrm{B}\left(p ; T_{i}\right)$ describes a projective line; we can assume without loss of generality that none of these three lines is the line at infinity. (If it is, we conjugate $T_{1}, T_{2}, T_{3}$ by an element of $\mathrm{PSL}_{2}(\mathbb{R})$, which is allowed since desired conclusion, Condition (2), is insensitive to conjugation.) Thus the determinant $\Delta(p)$ is defined - and, by assumption, vanishes - for all but finitely many values of $p$. Hence, in any expression $N(p) / D(p)$ of $\Delta(p)$ as a rational function of $p$, the numerator $N(p)$ is the zero polynomial. We will show, by analyzing the possible cases, that this implies the desired conclusion.

Case 1. One of $T_{i}$ (say $T_{1}$ ) is hyperbolic and another (say $T_{2}$ ) is not elliptic.

We first consider the case that the set $\operatorname{Fix}\left(T_{1}\right) \cap \operatorname{Fix}\left(T_{2}\right)$ is not empty. By conjugation, we can assume that $T_{1}$ fixes 0 and $\infty$, and $T_{2}$ fixes $\infty$. The matrix 
presentations in $\mathrm{SL}_{2}(\mathbb{R})$ of these elements are

$$
T_{1}=\left(\begin{array}{cc}
a_{1} & 0 \\
0 & 1 / a_{1}
\end{array}\right), \quad T_{2}=\left(\begin{array}{cc}
a_{2} & b_{2} \\
0 & 1 / a_{2}
\end{array}\right), \quad T_{3}=\left(\begin{array}{ll}
a_{3} & b_{3} \\
c_{3} & d_{3}
\end{array}\right)
$$

The constant term of $N$ is then $4 a_{2} b_{2} b_{3}\left(a_{2} b_{2} d_{3}-b_{3}\right)$. Since $a_{2}$ is not zero, we have either $b_{2}=0, b_{3}=0$ or $a_{2} b_{2} d_{3}=b_{3}$.

When $b_{2}=0$, the coefficient of $p^{2}$ is $-4 b_{3} d_{3}\left(a_{1}^{2}-a_{2}^{2}\right)\left(a_{2}^{2}-1\right)$. We have $T_{1}=T_{2}$ in $\mathrm{PSL}_{2}(\mathbb{R})$ when $a_{1}^{2}=a_{2}^{2}$, we have $T_{1}$ is trivial when $a_{2}^{2}=1$, and the case that $b_{3}=0$ will be discussed later. So $d_{3}$ is to be 0 , which together with $\operatorname{det} T_{3}=1$ implies $-b_{3} c_{3}=1$. The coefficient of $p^{3}$ is then $8\left(a_{1}^{2}-a_{2}^{2}\right)\left(a_{2}^{2}-1\right)$. All of the possible cases when it is zero have already been discussed.

When $b_{3}=0$, we have $d_{3}=1 / a_{3}$, for $\operatorname{det} T_{3}=1$. The coefficient of $p^{3}$ is then $8 a_{2} b_{2} c_{3}\left(a_{2}^{2} a_{3}^{2}-a_{1}^{2}\right) / a_{3}$. It is a straightforward calculation that there will be no new possible cases when either $b_{2}$ or $c_{3}$ is zero. We thus assume $a_{3}=a_{1} / a_{2}$ without loss of generality. The polynomial $N$ is then expressed as

$$
N(p)=\frac{4}{a_{2}}\left(\left(a_{1}^{2}-a_{2}^{2}\right)\left(a_{2}^{2}-1\right)+a_{1} a_{2}^{2} b_{2} c_{3}\right)\left(b_{2}-a_{1} c_{3} z^{2}\right) p^{2} .
$$

We thus have $c_{3}=\left(a_{1}^{2}-a_{2}^{2}\right)\left(1-a_{2}^{2}\right) /\left(a_{1} a_{2}^{2} b_{2}\right)$. The point $a_{2} b_{2} /\left(1-a_{2}^{2}\right)$ is fixed by $T_{2}$. After normalizing this fixed point to be 1 , three elements $T_{1}, T_{2}$ and $T_{3}$ coincide with the ones in $(2 b)$.

When $a_{2} b_{2} d_{3}=b_{3}$, we have $c_{3}=\left(a_{3} d_{3}-1\right) /\left(a_{2} b_{2} d_{3}\right)$, for $\operatorname{det} T_{3}=1$. The coefficient of $p$ is then $8 a_{2}{ }^{2} b_{2}{ }^{2}\left(a_{2}{ }^{2} d_{3}{ }^{2}-1\right)$, which implies $T_{2}=T_{3}$, contrary to assumption.

We next consider the case that $\operatorname{Fix}\left(T_{1}\right) \cap \operatorname{Fix}\left(T_{2}\right)$ is the empty set; here $T_{1}$ can be assumed to fix 0 and $\infty$, and $T_{2}$ to fix 1 . The matrix presentations in $\mathrm{SL}_{2}(\mathbb{R})$ of these elements are

$$
T_{1}=\left(\begin{array}{cc}
a_{1} & 0 \\
0 & \frac{1}{a_{1}}
\end{array}\right), \quad T_{2}=\left(\begin{array}{cc}
a_{2} & \frac{1}{a_{2}-c_{2}}-a_{2} \\
c_{2} & \frac{1}{a_{2}-c_{2}}-c_{2}
\end{array}\right), \quad T_{3}=\left(\begin{array}{cc}
a_{3} & \frac{a_{3} d_{3}-1}{c_{3}} \\
c_{3} & d_{3}
\end{array}\right) .
$$

The coefficient of $p^{6}$ is then $-4 a_{1}^{2} c_{2} c_{3}^{3}\left(a_{2}-c_{2}\right)^{2}\left(a_{2} c_{3}-a_{3} c_{2}\right)$. When it is zero, we have $a_{2} c_{3}-a_{3} c_{2}=0$, otherwise we are in the case already considered. This implies $T_{2}=T_{3}$.

Case 2. Two of $T_{i}$ (say $T_{1}$ and $T_{2}$ ) are parabolic.

We first consider the case that $\operatorname{Fix}\left(T_{1}\right)$ coincides with $\operatorname{Fix}\left(T_{2}\right)$; the common fixed point of $T_{1}$ and $T_{2}$ is assumed to be $\infty$. The matrix presentations in $\mathrm{SL}_{2}(\mathbb{R})$ of these 
elements are

$$
T_{1}=\left(\begin{array}{cc}
1 & b_{1} \\
0 & 1
\end{array}\right), \quad T_{2}=\left(\begin{array}{cc}
1 & b_{2} \\
0 & 1
\end{array}\right), \quad T_{3}=\left(\begin{array}{ll}
a_{3} & b_{3} \\
c_{3} & d_{3}
\end{array}\right)
$$

where $a_{3} d_{3}-b_{3} c_{3}=1$. It is then a straightforward calculation that there will be no new possible cases.

We next consider the case that the set $\operatorname{Fix}\left(T_{1}\right) \cap \operatorname{Fix}\left(T_{2}\right)$ is empty; $T_{1}$ is assumed to fix $\infty$ and $T_{2}$ is assumed to fix 0 . The matrix presentations in $\mathrm{SL}_{2}(\mathbb{R})$ of these elements are

$$
T_{1}=\left(\begin{array}{cc}
1 & b_{1} \\
0 & 1
\end{array}\right), \quad T_{2}=\left(\begin{array}{cc}
1 & 0 \\
c_{2} & 1
\end{array}\right), \quad T_{3}=\left(\begin{array}{cc}
a_{3} & b_{3} \\
\frac{a_{3} d_{3}-1}{b_{3}} & d_{3}
\end{array}\right) .
$$

The constant term is then $8 b_{3}{ }^{3}\left(b_{1} d_{3}-b_{3}\right)$, which implies $T_{1}=T_{3}$.

Case 3. Two of the $T_{i}$ are elliptic.

We assume $T_{1}$ fixes $\sqrt{-1}$ and $T_{2}$ fixes $h \sqrt{-1}$, for some $h>0$. The matrix presentations in $\mathrm{SL}_{2}(\mathbb{R})$ of these elements are

$$
T_{1}=\left(\begin{array}{cc}
\cos \theta_{1} & \sin \theta_{1} \\
-\sin \theta_{1} & \cos \theta_{1}
\end{array}\right), \quad T_{2}=\left(\begin{array}{cc}
\cos \theta_{2} & h \sin \theta_{2} \\
-\frac{1}{h} \sin \theta_{2} & \cos \theta_{2}
\end{array}\right), \quad T_{3}=\left(\begin{array}{ll}
a & b \\
c & d
\end{array}\right),
$$

where $\theta_{1}, \theta_{2} \in \mathbb{R}$ and $a d-b c=1$. Recall $\sin \theta_{i} \neq 0$ for $i=1,2$ since both $T_{1}$ and $T_{2}$ are not trivial.

Let $p_{21}:=h\left(-1+\cos \theta_{2}\right) / \sin \theta_{2}$ and $p_{22}:=h\left(1+\cos \theta_{2}\right) / \sin \theta_{2}$. These points satisfy $\sqrt{-1} \in \mathrm{B}\left(p_{2 i} ; T_{2}\right)$ for $i=1,2$. Since we are assuming (1), we also have $\sqrt{-1} \in \mathrm{B}\left(p_{2 i} ; T_{3}\right)$ for $i=1,2$, which means $\mathrm{C}\left(p_{2 i} ; T_{3}\right) \notin \mathbb{R}^{2} \subset \mathbb{R} \mathbb{P}^{2}$.

Let $D_{i}$ be the denominator of $\mathrm{C}\left(p_{2 i} ; T_{3}\right)$ for $i=1,2$. They then satisfy

$$
D_{1}-D_{2}=\frac{-4 h}{\sin ^{2} \theta_{2}} d_{1}, \quad D_{1}+D_{2}=\frac{4 h \cos \theta_{2}}{\sin ^{2} \theta_{2}} d_{1}+2 d_{2},
$$

where $d_{1}:=(a b+c d) \sin \theta_{2}+h\left(a^{2}+c^{2}-1\right) \cos \theta_{2}$ and $d_{2}:=h^{2}\left(a^{2}+c^{2}-1\right)+$ $\left(b^{2}+d^{2}-1\right)$. Since $\mathrm{C}\left(p_{2 i} ; T_{3}\right) \notin \mathbb{R}^{2}$, we have $D_{1}=D_{2}=0$. This implies, by the equations above, $d_{1}=d_{2}=0$.

Similarly, there are points $p_{1 i}$ in $\partial \mathbb{U}^{2}$ satisfying $h \sqrt{-1} \in \mathrm{B}\left(p_{1 i} ; T_{1}\right)$ for $i=1,2$. Let

$$
H:=\left(\begin{array}{cc}
\sqrt{h} & 0 \\
0 & 1 / \sqrt{h}
\end{array}\right) .
$$

We then have $\operatorname{Fix}\left(H^{-1} T_{1} H\right)=\{\sqrt{-1} / h\}$ and $\operatorname{Fix}\left(H^{-1} T_{2} H\right)=\{\sqrt{-1}\}$. Applying similar calculations as before we have $d_{3}=d_{4}=0$, where 


$$
\begin{aligned}
& d_{3}:=(a b+c d h) \sin \theta_{1}+\left(a^{2}+c^{2} h^{2}-1\right) \cos \theta_{1}, \\
& d_{4}:=\left(a^{2}+b^{2}-1\right)+\left(c^{2}+d^{2}-1\right) h^{2} .
\end{aligned}
$$

When $c=0$, we have $a=1 / d$ and $b=h\left(d^{2}-1\right) \cos \theta_{2} /\left(d \sin \theta_{2}\right)$. Substitute them for both $d_{2}$ and $d_{4}$ and we have $d_{4}-d_{2}=\left(d^{4}-1\right)\left(h^{2}-1\right) \sin ^{2} \theta_{2}$. The condition $d_{4}-d_{2}=0$ implies that $T_{3}$ is trivial.

When $c \neq 0$, we have $b=(a d-1) / c$. Substitute it for both $d_{2}$ and $d_{4}$ and we have $d_{2}-d_{4}=c^{2}\left(a^{2}-d^{2}\right)\left(h^{2}-1\right)$. When $h^{2}=1$ or $a=d$, a straightforward calculation shows that $T_{1}, T_{2}$ and $T_{3}$ have a common fixed point. When $a=-d$, we have the following expression of $d_{1}$ :

$$
d_{1}=-a\left(a^{2}+c^{2}+1\right) \sin \theta_{2}+c h\left(a^{2}+c^{2}-1\right) \cos \theta_{2} .
$$

Suppose $a^{2}+c^{2}-1 \neq 0$, otherwise we have no new triple. Then $h$ is expressed as

$$
h=\frac{a\left(a^{2}+c^{2}+1\right) \sin \theta_{2}}{c\left(a^{2}+c^{2}-1\right) \cos \theta_{2}} .
$$

Substitute them for $d_{2}$ and we have the expression of $\sin ^{2} \theta_{2}$ as

$$
\sin ^{2} \theta_{2}=\frac{\left(a^{2}+c^{2}-1\right)\left(\left(a^{2}+c^{2}-1\right)\left(a^{2}-1\right)+4 a^{2}\right)}{4 c^{2}-\left(a^{2}+c^{2}+1\right)} .
$$

Similarly, substitute them for $d_{3}$ and we have the expression of $\sin ^{2} \theta_{1}$ as

$$
\sin ^{2} \theta_{1}=\frac{(2 a c)^{2}}{\left(a^{2}+c^{2}+1\right)-4 c^{2}} .
$$

These expressions imply, after a few more calculations, that there will be no new triple in this case. This concludes the proof of Proposition 2.

Proposition 3. For $i=1,2$, let $\mathrm{B}_{i}$ be the perpendicular bisector of the hyperbolic line segment $\left[z_{0}, z_{i}\right]$ with endpoints $z_{0}$ and $z_{i}$ in $\mathbb{H}^{2}$, and $\mathrm{C}_{i}$ the pole in $\mathbb{R} \mathbb{P}^{2}$ of the projective line containing the image of $\mathrm{B}_{i}$. In each of the following triples of statement, the three conditions are equivalent:

(1-1) The points $z_{0}, z_{1}$ and $z_{2}$ are on a hyperbolic circle with center $w \in \mathbb{U}^{2}$.

(1-2) The projective lines $\mathrm{B}_{1}$ and $\mathrm{B}_{2}$ intersect at $w \in \mathbb{H}^{2}$.

(1-3) The projective line in $\mathbb{R P}^{2}$ through $\mathrm{C}_{1}$ and $\mathrm{C}_{2}$ does not intersect $\overline{D^{2}}$, and its pole corresponds to $w \in \mathbb{H}^{2}$.

(2-1) The points $z_{0}, z_{1}$ and $z_{2}$ are on a horocycle with center $w \in \partial \mathbb{H}^{2}$.

(2-2) The projective lines $\overline{\mathrm{B}_{1}}$ and $\overline{\mathrm{B}_{2}}$ intersect at $w \in \partial \mathbb{M}^{2}$.

(2-3) The projective line in $\mathbb{R} \mathbb{P}^{2}$ through $\mathrm{C}_{1}$ and $\mathrm{C}_{2}$ touches $\partial D^{2}$, and the tangential point corresponds to $w \in \partial \mathbb{Q}^{2}$. 
(3-1) The points $z_{0}, z_{1}$ and $z_{2}$ are on an equidistant point set whose axis is $\ell$.

(3-2) The projective lines $\overline{\mathrm{B}_{1}}$ and $\overline{\mathrm{B}_{2}}$ intersect perpendicularly with $\ell$.

(3-3) The projective line in $\mathbb{R} \mathbb{P}^{2}$ through $\mathrm{C}_{1}$ and $\mathrm{C}_{2}$ intersects with $D^{2}$, and it contains the image of $\ell$.

Proof. For $j=1,2,3$, the equivalence between $(j-2)$ and $(j-3)$ comes from the duality for the pole and the projective line in $\mathbb{R P}^{2}$.

Suppose that (1-1) holds. Since any point on $\mathrm{B}_{1}$ is equidistant from $z_{0}$ and $z_{1}$, the center $w$ lies on $\mathrm{B}_{1}$, and so does on $\mathrm{B}_{2}$. Thus (1-3) holds, and the converse is also true by the same argument. The equivalence between (2-1) and (2-2) comes by a continuity argument from the equivalence above. The equivalence between (3-1) and (3-2) comes from Proposition 1 (3).

Lemma 4. Let $T_{1}, T_{2}$ and $T_{3}$ be mutually distinct and nontrivial elements in a Fuchsian group. Suppose that three points $\mathrm{C}\left(p ; T_{1}\right), \mathrm{C}\left(p ; T_{2}\right)$ and $\mathrm{C}\left(p ; T_{3}\right)$ are collinear in $\mathbb{R} P^{2}$ for any $p$ in $\partial \mathbb{H}^{2}-\bigcup_{i=1}^{3} \operatorname{Fix}\left(T_{i}\right)$, and that there is a point $p_{0}$ in $\partial \mathbb{I}^{2}-\bigcup_{i=1}^{3} \operatorname{Fix}\left(T_{i}\right)$ such that the set

$$
\mathrm{B}\left(p_{0} ; T_{1}\right) \cap \mathrm{B}\left(p_{0} ; T_{2}\right) \cap \mathrm{B}\left(p_{0} ; T_{3}\right) \quad\left(\text { resp. } \overline{\mathrm{B}\left(p_{0} ; T_{1}\right)} \cap \overline{\mathrm{B}\left(p_{0} ; T_{2}\right)} \cap \overline{\mathrm{B}\left(p_{0} ; T_{3}\right)}\right)
$$

is not empty. Then $T_{1}, T_{2}$ and $T_{3}$ belong to a cyclic elliptic (resp. parabolic) subgroup.

Proof. Suppose that $\mathrm{C}\left(p ; T_{1}\right), \mathrm{C}\left(p ; T_{2}\right)$ and $\mathrm{C}\left(p ; T_{3}\right)$ are collinear in $\mathbb{R} P^{2}$ for any $p$ in $\partial \mathbb{H}^{2}-\bigcup_{i=1}^{3} \operatorname{Fix}\left(T_{i}\right)$. Since Proposition $2(2 \mathrm{~b})$ does not occur from elements in a Fuchsian group, the elements $T_{1}, T_{2}$ and $T_{3}$ have the same fixed point set. Furthermore, they belong to a cyclic subgroup generated by, say $T$, since they come from a Fuchsian group.

We first consider the case that $T$ is hyperbolic; in particular, the axis of $T$ is assumed to be contained in the imaginary axis of $\mathbb{C}$. The circle in $\widehat{\mathbb{C}}$ through $p, T_{1}(p), T_{2}(p)$ and $T_{3}(p)$ is a Euclidean line through the origin for any $p$ in $\partial \mathbb{\boxplus}^{2}-\{0, \infty\}$. Then the hyperbolic lines $\mathrm{B}\left(p ; T_{i}\right)$ are ultraparallel by Proposition 3 so that there is no such point $p_{0}$ in question.

We next consider the case that $T$ is parabolic; in particular, $T$ is assumed to fix $\infty$. For each $i=1,2,3$, the hyperbolic line $\mathrm{B}\left(p ; T_{i}\right)$ is contained in a vertical Euclidean line for any $p$ in $\partial \mathbb{Q}^{2}-\{\infty\}$. The set

$$
\overline{\mathrm{B}\left(p ; T_{1}\right)} \cap \overline{\mathrm{B}\left(p ; T_{2}\right)} \cap \overline{\mathrm{B}\left(p ; T_{3}\right)}
$$

then coincides with $\{\infty\}$.

Finally, if $T$ is elliptic, then the four points are on a hyperbolic circle centered at the fixed point of $T$ for any $p$ in $\partial \mathbb{\boxplus}^{2}$. 
Proposition 5. Let $T_{1}, T_{2}$ and $T_{3}$ be elements in a Fuchsian group. Suppose that they are mutually distinct, nontrivial and neither elliptic with a common fixed point nor parabolic with a common fixed point. Then $\mathscr{V}_{T_{1}, T_{2}, T_{3}}$ has measure zero.

Proof. We first consider the case that $T_{1}, T_{2}$ and $T_{3}$ belong to a cyclic subgroup of a given Fuchsian group $\Gamma$. By the assumption, the subgroup is generated by a hyperbolic element $T$. By Proposition 1, the set $\mathscr{V}_{T_{1}, T_{2}, T_{3}}$ coincides with the fixed point set $\operatorname{Fix}(T)$ of $T$, which consists of two points. So $\mathscr{V}_{T_{1}, T_{2}, T_{3}}$ has measure zero.

We next consider the case that $T_{1}, T_{2}$ and $T_{3}$ do not belong to any cyclic subgroup of $\Gamma$. Define

$$
\mathscr{V}_{T_{1}, T_{2}, T_{3}}^{\prime}:=\left\{p \in \partial \mathbb{R}^{2} \mid \mathrm{C}\left(p ; T_{1}\right), \mathrm{C}\left(p ; T_{2}\right) \text { and } \mathrm{C}\left(p ; T_{3}\right) \text { are collinear in } \mathbb{R} P^{2}\right\} .
$$

As is mentioned in the proof of Proposition 2, the set $\mathscr{V}_{T_{1}, T_{2}, T_{3}}^{\prime}$ is the solution set of a real algebraic equation with variable $p$. By the assumption together with Proposition 2, the equation is proper. So $\mathscr{V}_{T_{1}, T_{2}, T_{3}}^{\prime}$ has measure zero in $\partial \mathbb{M}^{2}$. By Proposition 3 the set $\mathscr{V}_{T_{1}, T_{2}, T_{3}}$ is contained in $\mathscr{V}_{T_{1}, T_{2}, T_{3}}^{\prime}$; when the projective line through $\mathrm{C}\left(p ; T_{1}\right), \mathrm{C}\left(p ; T_{2}\right)$ and $\mathrm{C}\left(p ; T_{3}\right)$ intersects with $D^{2}$, the point $p$ is not contained in $\mathscr{V}_{T_{1}, T_{2}, T_{3}}$. The set $\mathscr{V}_{T_{1}, T_{2}, T_{3}}$ then has measure zero in $\partial \mathbb{\boxplus}^{2}$ as well.

Lemma 6. Let $T_{1}$ and $T_{2}$ be distinct and nontrivial elements in $\mathrm{PSL}_{2}(\mathbb{R})$. Suppose that they are not parabolic with a common fixed point. Then there is a point $p_{0}$ in $\partial \mathbb{U}^{2}-\left(\operatorname{Fix}\left(T_{1}\right) \cup \operatorname{Fix}\left(T_{2}\right)\right)$ such that $\overline{\mathrm{B}\left(p_{0} ; T_{1}\right)}$ and $\overline{\mathrm{B}\left(p_{0} ; T_{2}\right)}$ do not share their endpoints.

Proof. We first consider the case that both $T_{1}$ and $T_{2}$ are elliptic. Take the hyperbolic line $\ell$ through their fixed points (choose any hyperbolic line which contains the fixed point if their fixed points are identical). Then there is a point $p_{0}$ in $\partial \mathbb{U}^{2}$ such that $\ell$ coincides with $\mathrm{B}\left(p_{0} ; T_{1}\right)$. On the other hand, $\mathrm{B}\left(p_{0} ; T_{2}\right)$ always contains the fixed point of $T_{2}$ by Proposition 1 . So $\mathrm{B}\left(p_{0} ; T_{2}\right)$ intersects with $\mathrm{B}\left(p_{0} ; T_{1}\right)=\ell$ at the fixed point of $T_{2}$. If $\ell$ happen to coincide with $\mathrm{B}\left(p_{0} ; T_{2}\right)$, then take another point $p_{0}$ satisfying the same condition, or switch $T_{1}$ and $T_{2}$ and do the same argument. Then you can find a point in question on $\partial \mathbb{U}^{2}$ since $T_{1}$ and $T_{2}$ are different.

We next consider the case that $T_{1}$ is elliptic and that $T_{2}$ is either parabolic or hyperbolic. For any point in $\mathbb{\boxplus}^{2}-\operatorname{Fix}\left(T_{2}\right)$, there is $p$ in $\partial \mathbb{U}^{2}-\operatorname{Fix}\left(T_{2}\right)$ such that $\mathrm{B}\left(p ; T_{2}\right)$ contains the point. So there is a point $p_{0}$ in $\partial \mathbb{Q}^{2}$ such that $\mathrm{B}\left(p_{0} ; T_{2}\right)$ contains the fixed point of $T_{1}$, which is on $\mathrm{B}\left(p_{0} ; T_{1}\right)$ since $T_{1}$ is elliptic.

We then consider the case that both $T_{1}$ and $T_{2}$ are not elliptic. Suppose first that their fixed point sets are different. Let $p_{1}$ be a fixed point of $T_{1}$ which is not fixed by $T_{2}$, for example. Since $T_{1}$ and $T_{2}$ are not elliptic, $\mathrm{B}\left(p ; T_{1}\right)$ converges to $p_{1}$ when $p$ approaches to $p_{1}$. A point $p$ close enough to $p_{1}$ is mapped to another point $T_{2}(p)$ by $T_{2}$, which is not close to $p_{1}$. Then the endpoints of $\overline{\mathrm{B}\left(p ; T_{2}\right)}$ are 
not close to $p_{1}$ as well, which means that $\overline{\mathrm{B}\left(p ; T_{1}\right)}$ and $\overline{\mathrm{B}\left(p ; T_{2}\right)}$ do not share their endpoints.

If $T_{1}$ and $T_{2}$ are not elliptic and their fixed point sets coincide with each other, then they are hyperbolic by the assumption. Then $\overline{\mathrm{B}\left(p ; T_{1}\right)}$ and $\overline{\mathrm{B}\left(p ; T_{2}\right)}$ are ultraparallel for any $p$ in $\partial \mathbb{\boxplus}^{2}-\left(\operatorname{Fix}\left(T_{1}\right) \cup \operatorname{Fix}\left(T_{2}\right)\right)$ by Proposition 3 .

Proposition 7. Let $T_{1}$ and $T_{2}$ be distinct and nontrivial elements in $\mathrm{PSL}_{2}(\mathbb{R})$. Suppose that they are not parabolic with a common fixed point. Then $\mathscr{T}_{T_{1}, T_{2}}$ has measure zero.

Proof. The set $\mathscr{T}_{T_{1}, T_{2}}$ is defined as the solution set of an algebraic equation. Actually it is given by calculating the Euclidean distance between the origin in $D^{2}$ and the projective line through $\mathrm{C}\left(p ; T_{1}\right)$ and $\mathrm{C}\left(p ; T_{2}\right)$. See [Jørgensen and Marden 1988, Lemma 3.3]. Lemma 6 means that the equation is proper. So $\mathscr{T}_{T_{1}, T_{2}}$ has measure zero.

Lemma 8. Let $T$ be a nontrivial element in $\mathrm{PSL}_{2}(\mathbb{R})$. If there is a point $w$ in $\overline{\mathbb{H}^{2}}$ such that $\overline{\mathrm{B}(p ; T)}$ contains $w$ for any $p$ in $\partial \mathbb{U}^{2}-\operatorname{Fix}(T)$, then $T$ is either elliptic or parabolic and $w$ is its fixed point. Similarly, for a hyperbolic line $\ell$, if $\mathrm{B}(p ; T)$ intersects perpendicularly with $\ell$ for any $p$ in $\partial \mathbb{U}^{2}-\operatorname{Fix}(T)$, then $T$ is hyperbolic and $\ell$ is its axis.

Proof. Suppose that $T$ fixes $w$. Then the statement holds only if $T$ is elliptic or parabolic by Proposition 1 .

Suppose that $T$ does not fix $w$. If $T$ is elliptic, there is a point $p_{0}$ in $\partial \mathbb{U}^{2}$ such that $\mathrm{B}\left(p_{0} ; T\right)$ does not contain $w$, for the fixed point of $T$ is the unique point that lies in $\mathrm{B}(p ; T)$ for any $p$ in $\partial \mathbb{Z}^{2}$. If $T$ is parabolic, then $\overline{\mathrm{B}(p ; T)}$ does not contain $w$ for any point $p$ close enough to the fixed point of $T$, for $\overline{\mathrm{B}(p ; T)}$ converges to the fixed point. The same argument holds when $T$ is hyperbolic.

For a hyperbolic line $\ell$, consider its image in $D^{2}$. Let $\mathrm{C}_{\ell}$ be the pole of the image of $\ell$. Then we interpret the assumption as the projective line $L_{p}$ containing the image of $\mathrm{B}(p ; T)$ contains $\mathrm{C}_{\ell}$ for any $p$ in $\partial \mathbb{H}^{2}-\operatorname{Fix}(T)$. If $T$ is elliptic or parabolic, then $L_{p}$ contains the image of its fixed point for any $p$ in $\partial \mathbb{U}^{2}-\operatorname{Fix}(T)$. If $T$ is hyperbolic, then $L_{p}$ contains the pole of the image of its axis for any $p$ in $\partial \mathbb{H}^{2}-\operatorname{Fix}(T)$. This implies that, if the assumption holds, then $T$ is hyperbolic with axis $\ell$.

Proposition 9. Let $T_{1}$ and $T_{2}$ be distinct and nontrivial elements in $\mathrm{PSL}_{2}(\mathbb{R})$. Suppose that $\operatorname{Fix}\left(T_{1}\right)$ and $\operatorname{Fix}\left(T_{2}\right)$ are different. Then $\mathscr{F} T_{1}, T_{2}$ has measure zero.

Proof. Let $z_{1}$ be a point in $\mathbb{R P}^{2}$ which corresponds to the fixed point of $T_{1}$ if it is elliptic or parabolic, or to the pole of the axis of $T_{1}$ if it is hyperbolic. We denote by $\ell_{2}$ the projective line in $\mathbb{R P}^{2}$ containing the image of $\mathrm{B}\left(p ; T_{2}\right)$. Then $\mathscr{F}_{T_{1}, T_{2}}$ is 
interpreted as

$$
{ }^{\mathscr{F}} T_{1}, T_{2}=\left\{p \in \partial \mathbb{T}^{2} \mid \ell_{2} \text { contains } z_{1}\right\} .
$$

Using this interpretation, the set $\mathscr{F}_{T_{1}}, T_{2}$ is defined as the solution set of an algebraic equation. To see it, use the Minkowski space model. The equation is given by the Minkowski inner product of $p_{1}$ and the normal vector of $\ell_{2}$. Lemma 8 means that this algebraic equation is proper. So the set $\mathscr{F}_{T} T_{1}, T_{2}$ has measure zero.

\section{Acknowledgement}

The author thanks Professor Raquel Díaz and Professor Makoto Sakuma for fruitful discussion on this research. The author is grateful to the hospitality of Professor Caroline Series and the University of Warwick, and Professor Albert Marden and the University of Minnesota. Thank you also to the referee for his/her valuable suggestions.

\section{References}

[Bacher and Vdovina 2002] R. Bacher and A. Vdovina, "Counting 1-vertex triangulations of oriented surfaces”, Discrete Math. 246:1-3 (2002), 13-27. MR 2003d:05106 Zbl 0994.05058

[Beardon 1983] A. F. Beardon, The geometry of discrete groups, Graduate Texts in Mathematics 91, Springer, New York, 1983. MR 85d:22026 Zbl 0528.30001

[Díaz and Ushijima 2009] R. Díaz and A. Ushijima, "On the properness of some algebraic equations appearing in Fuchsian groups", Topology Proc. Auburn Univ. 33 (2009), 81-106. MR 2010i:30056 Zbl 1179.30039

[Fricke and Klein 1897] R. Fricke and F. Klein, Vorlesungen über die Theorie der automorphen Functionen. Erster Band; Die gruppentheoretischen Grundlagen, B. G. Teubner, Leipzig, 1897.

[Girondo and Nakamura 2007] E. Girondo and G. Nakamura, "Compact non-orientable hyperbolic surfaces with an extremal metric disc", Conform. Geom. Dyn. 11 (2007), 29-43. MR 2007k:30087 Zbl 1185.30047

[Jørgensen and Marden 1988] T. Jørgensen and A. Marden, "Generic fundamental polyhedra for Kleinian groups", pp. 69-85 in Holomorphic functions and moduli, II (Berkeley, 1986), edited by D. Drasin, Math. Sci. Res. Inst. Publ. 11, Springer, New York, 1988. MR 89h:30061 Zbl 0673.30034

[Jørgensen and Näätänen 1982] T. Jørgensen and M. Näätänen, "Surfaces of genus 2: generic fundamental polygons", Quart. J. Math. Oxford Ser. (2) 33:132 (1982), 451-461. MR 84c:51029

[Keen 1966] L. Keen, "Canonical polygons for finitely generated Fuchsian groups", Acta Math. 115 (1966), 1-16. MR 32 \#1349 Zbl 0144.34101

[Näätänen 1985] M. Näätänen, "On the stability of identification patterns for Dirichlet regions", Ann. Acad. Sci. Fenn. Ser. A I Math. 10 (1985), 411-417. MR 86k:30054

[Näätänen and Penner 1991] M. Näätänen and R. C. Penner, "The convex hull construction for compact surfaces and the Dirichlet polygon", Bull. London Math. Soc. 23:6 (1991), 568-574. MR 93c:30056

[Nakamura 2004] G. Nakamura, "Generic fundamental polygons for surfaces of genus three", Kodai Math. J. 27:1 (2004), 88-104. MR 2005a:30069 Zbl 1059.30032 
[Vdovina 2008] A. Vdovina, "On the number of optimal surfaces", pp. 557-567 in The Zieschang Gedenkschrift, edited by M. Boileau et al., Geom. Topol. Monogr. 14, Geom. Topol., Coventry, 2008. MR 2010a:53055 Zbl 1138.53037

Received May 6, 2010. Revised February 16, 2011.

\author{
AKIRA USHIJIMA \\ FACULTY OF MATHEMATICS AND PHYSICS \\ INSTITUTE OF SCIENCE AND ENGINEERING \\ KANAZAWA UNIVERSITY \\ KANAZAWA 920-1192 \\ JAPAN \\ ushijima@kenroku.kanazawa-u.ac.jp
}




\title{
PACIFIC JOURNAL OF MATHEMATICS
}

\author{
http://www.pjmath.org \\ Founded in 1951 by
}

E. F. Beckenbach (1906-1982) and F. Wolf (1904-1989)

\section{EDITORS}

V. S. Varadarajan (Managing Editor)

Department of Mathematics

University of California

Los Angeles, CA 90095-1555

pacific@math.ucla.edu

Vyjayanthi Chari

Department of Mathematics

University of California

Riverside, CA 92521-0135

chari@math.ucr.edu

\section{Robert Finn}

Department of Mathematics Stanford University

Stanford, CA 94305-2125

finn@math.stanford.edu

Kefeng Liu

Department of Mathematics

University of California

Los Angeles, CA 90095-1555

liu@math.ucla.edu
Darren Long

Department of Mathematics

University of California

Santa Barbara, CA 93106-3080

long@math.ucsb.edu

Jiang-Hua Lu

Department of Mathematics

The University of Hong Kong

Pokfulam Rd., Hong Kong jhlu@maths.hku.hk

Alexander Merkurjev

Department of Mathematics University of California

Los Angeles, CA 90095-1555 merkurev@math.ucla.edu
Sorin Popa

Department of Mathematics

University of California

Los Angeles, CA 90095-1555

popa@math.ucla.edu

Jie Qing

Department of Mathematics

University of California

Santa Cruz, CA 95064

qing@ cats.ucsc.edu

Jonathan Rogawski

Department of Mathematics

University of California

Los Angeles, CA 90095-1555

jonr@math.ucla.edu

\section{PRODUCTION}

pacific@math.berkeley.edu

Silvio Levy, Scientific Editor Matthew Cargo, Senior Production Editor

ACADEMIA SINICA, TAIPEI

CALIFORNIA INST. OF TECHNOLOGY

INST. DE MATEMÁTICA PURA E APLICADA

KEIO UNIVERSITY

MATH. SCIENCES RESEARCH INSTITUTE

NEW MEXICO STATE UNIV.

OREGON STATE UNIV.

\section{SUPPORTING INSTITUTIONS}

STANFORD UNIVERSITY
UNIV. OF BRITISH COLUMBIA
UNIV. OF CALIFORNIA, BERKELEY
UNIV. OF CALIFORNIA, DAVIS
UNIV. OF CALIFORNIA, LOS ANGELES
UNIV. OF CALIFORNIA, RIVERSIDE
UNIV. OF CALIFORNIA, SAN DIEGO
UNIV. OF CALIF., SANTA BARBARA

UNIV. OF CALIF., SANTA CRUZ

UNIV. OF MONTANA

UNIV. OF OREGON

UNIV. OF SOUTHERN CALIFORNIA

UNIV. OF UTAH

UNIV. OF WASHINGTON

WASHINGTON STATE UNIVERSITY

These supporting institutions contribute to the cost of publication of this Journal, but they are not owners or publishers and have no responsibility for its contents or policies.

See inside back cover or www.pjmath.org for submission instructions.

The subscription price for 2011 is US \$420/year for the electronic version, and \$485/year for print and electronic.

Subscriptions, requests for back issues from the last three years and changes of subscribers address should be sent to Pacific Journal of Mathematics, P.O. Box 4163, Berkeley, CA 94704-0163, U.S.A. Prior back issues are obtainable from Periodicals Service Company, 11 Main Street, Germantown, NY 12526-5635. The Pacific Journal of Mathematics is indexed by Mathematical Reviews, Zentralblatt MATH, PASCAL CNRS Index, Referativnyi Zhurnal, Current Mathematical Publications and the Science Citation Index.

The Pacific Journal of Mathematics (ISSN 0030-8730) at the University of California, c/o Department of Mathematics, 969 Evans Hall, Berkeley, CA 94720-3840, is published monthly except July and August. Periodical rate postage paid at Berkeley, CA 94704, and additional mailing offices. POSTMASTER: send address changes to Pacific Journal of Mathematics, P.O. Box 4163, Berkeley, CA 94704-0163.

PJM peer review and production are managed by EditFLOW ${ }^{\mathrm{TM}}$ from Mathematical Sciences Publishers.

PUBLISHED BY PACIFIC JOURNAL OF MATHEMATICS

at the University of California, Berkeley 94720-3840

A NON-PROFIT CORPORATION

Typeset in IATEX

Copyright $(2011$ by Pacific Journal of Mathematics 


\section{PACIFIC JOURNAL OF MATHEMATICS}

Volume $251 \quad$ No. $2 \quad$ June 2011

Two Kazdan-Warner-type identities for the renormalized volume coefficients and the Gauss-Bonnet curvatures of a Riemannian metric

BIN GUO, ZHENG-CHAO HAN and HAIZHONG LI

Gonality of a general ACM curve in $\mathbb{P}^{3}$

ROBIN HARTSHORNE and ENRICO SCHLESINGER

Universal inequalities for the eigenvalues of the biharmonic operator on submanifolds

SAÏD ILIAS and OLA MAKHOUL

Multigraded Fujita approximation

SHIN-YaO Jow

Some Dirichlet problems arising from conformal geometry

QI-RUi Li and WEIMIN SHENG

Polycyclic quasiconformal mapping class subgroups

KATSUHIKO MATSUZAKI

On zero-divisor graphs of Boolean rings

ALI MOHAMMADIAN

Rational certificates of positivity on compact semialgebraic sets

VICTORIA POWERS

Quiver grassmannians, quiver varieties and the preprojective algebra

Alistair Savage and Peter Tingley

Nonautonomous second order Hamiltonian systems

\section{MARTIN SCHECHTER}

Generic fundamental polygons for Fuchsian groups

AKIRA USHIJIMA

Stability of the Kähler-Ricci flow in the space of Kähler metrics

KAI ZHENG

The second variation of the Ricci expander entropy 